\title{
CARACTERÍSTICAS EPIDEMIOLÓGICAS, CLÍNICAS E LABORATORIAIS DE GATOS COM SINAIS DE DOENÇA DO TRATO URINÁRIO INFERIOR
}

\author{
Guadalupe Sampaio Ferreira ${ }^{1}$, Marileda Bonafim Carvalho', Michelle Lopes Avante ${ }^{1}$ \\ 1 UNESP - Jaboticabal \\ Correspondência: Guadalupe Sampaio Ferreira: ferreira.guadalupe@gmail.com
}

\begin{abstract}
RESUMO: A cistite idiopática felina (CIF) é a causa mais comum de doença do trato urinário inferior felino (DTUIF), enquanto a cistite bacteriana é diagnosticada somente em $1 \%$ a $3 \%$ dos casos. $O$ objetivo deste estudo foi avaliar gatos com apresentação ou histórico de sinais de doença do trato urinário inferior (SDTUI), considerando aspectos epidemiológicos, clínicos e laboratoriais. Cinco gatos sadios e 45 gatos com SDTUI foram avaliados no período de março de 2011 a outubro de 2012, por meio de histórico, exame físico, ultrassonografia abdominal e exames laboratoriais (hematológico, urinálise, bioquímica sérica e urinária e urocultura). Os diagnósticos dos gatos com SDTUI incluíram CIF não obstrutiva, CIF obstrutiva e cistite bacteriana. Não houve diferença significativa quanto à densidade, $\mathrm{pH}$ e qualidade do sedimento urinário entre as diferentes categorias etiológicas de DTUIF. A hematúria foi maior em gatos com SDTUI, e a piúria foi associada principalmente com cistite bacteriana. Adicionalmente, outros achados sugerem que raças específicas, idade, gênero e castração não estão associados com as manifestações de SDTUI em felinos.
\end{abstract}

Palavras-chave: gato; cistite; trato urinário inferior felino

\section{EPIDEMIOLOGIC, CLINIC AND LABORATORIAL CHARACTERISTICS OF DISEASE CATS WITH LOWER URINARY TRACT SIGNS}

\begin{abstract}
Feline idiopathic cystitis (FIC) is the most common cause of feline lower urinary tract disease (FLUTD), whereas bacterial cystitis is diagnosed only in $1 \%$ to $3 \%$ of the cases. The aim of this study was to evaluate cats with presentation or history of lower urinary tract signs disease (LUTSD), considering epidemiological, clinical and laboratory aspects. From March 2011 to October 2012, 45 cats presenting LUTSD and five health cats were evaluated regarding to the history, physical examination, abdominal ultrasonography and laboratorial analyses (CBC, urinalysis, clinical chemistry and urine culture). For the 45 cats presenting LUTSD, the diagnosis included nonobstructive FIC, obstructive FIC, and bacterial cystitis. There were no significant differences in the urine specific gravity, $\mathrm{pH}$ and quality of the urine sediment between al FLUTD categories. The hematuria was higher in cats with LUTSD, and piuria was associated mainly to bacterial cystitis. Additionally, other findings suggest that features as breed, age, gender, and neutering were not associated with LUTSD presentation in felines.
\end{abstract}

Key Words: cat; cystitis; feline lower urinary tract 


\section{INTRODUÇÃO}

Dentre as afecções mais frequentes dos felinos domésticos destacam-se as que acometem o trato urinário inferior. Independentemente da causa envolvida, as manifestações perceptíveis, inclusive pelos proprietários, compõem um conjunto único de sinais que podem ocorrer de forma isolada ou combinada. Estes são os "sinais de doença do trato urinário inferior" (SDTUI) que compreendem disúria, polaquiúria, hematúria, estrangúria, periúria e mudança de comportamento (WESTROPP, 2011).

O termo "doença idiopática do trato urinário inferior dos felinos" (DITUIF), diferentemente da denominação DTUIF, tem sido empregado para qualificar as formas idiopáticas, obstrutivas ou não obstrutivas, uma vez que a causa, ou causas continuam desconhecidas. $O$ uso do termo "cistite idiopática felina" (CIF) vem tomando o lugar da denominação DITUIF. Em função das semelhanças existentes entre a CIF e a cistite intersticial $(\mathrm{Cl})$ que acomete seres humanos, alguns têm empregado o termo $\mathrm{Cl}$ para fazer referência à $\mathrm{CIF}$ (BUFFINGTON, 1994; OSBORNE et al., 1999; KALKSTEIN, 2004; WESTROPP, 2011).

$\mathrm{Na}$ rotina, em clínicas brasileiras, ainda prevalecem as condutas de utilização mínima de exames complementares, se algum, e instituição de tratamento com antibiótico e dieta terapêutica para prevenção da formação de urólitos de todos os gatos com SDTUI. Com base na hipótese de que estudos clínicos nacionais possam contribuir para a prática da urologia felina, foi elaborado o presente trabalho cujo objetivo foi avaliar clínica e laboratorialmente gatos com sinais de doença do trato urinário inferior, qualificar os casos e investigar a existência de infecção bacteriana.

\section{MATERIAL E MÉTODOS}

Foi realizado nas dependências do Serviço de Nefrologia e Urologia Veterinária (SNUV), Laboratório de Nefrologia e Urologia do Grupo de Pesquisa em Nefrologia e Urologia Veterinária (GPNUV) e Laboratório de Patologia Clínica Veterinária, localizados no Hospital Veterinário (HV) da Faculdade de Ciências Agrárias e Veterinárias - Universidade Estadual Paulista - UNESP - campus de Jaboticabal e no Laboratório de Análises Clínicas São Lucas - Jaboticabal - SP (uroculturas).

O estudo seguiu um desenho epidemiológico descritivo de gatos, com ou sem sinais de doença do trato urinário inferior (SDTUI), atendidos no SNUV. Para compor os grupos foram avaliados gatos, machos ou fêmeas, encaminhados em função de apresentação de SDTUI ou para complementação de check-up em andamento.

Foram feitas avaliações pontuais dos aspectos clínicos e laboratoriais relacionados (resenha, anamnese, exame físico, hemograma, bioquímica sérica - ureia e creatinina, bioquímica urinária - creatinina e proteína, e urinálise), caracterização ultrassonográfica da vesícula urinária e urocultura. Todos os exames e coleta de amostras foram realizados no mesmo dia. $\mathrm{E}$ os procedimentos laboratoriais foram iniciados imediatamente após as coletas das amostras (sangue e urina). Os resultados das avaliações realizadas em outras ocasiões, visando ao atendimento completo do paciente, não foram incluídos no estudo. Contudo, todos os dados foram considerados na conclusão diagnóstica para inclusão ou exclusão dos pacientes no grupo estudado.

Foram estudados 50 gatos, sem restrição de raça ou sexo e com no mínimo um ano de idade. De acordo 
com as conclusões diagnósticas os casos foram distribuídos em quatro grupos, como se segue.

Grupo normal (GN): Composto por cinco gatos machos hígidos e sem histórico de doenças anteriores.

Grupo de cistite idiopática felina (GCIF): Composto por 28 gatos (19 machos e nove fêmeas), com histórico de DTUIF na forma não obstrutiva (cistite idiopática felina).

Grupo de cistite idiopática felina obstrutiva (GCIFO): Composto por 10 gatos machos, com histórico de DTUIF na forma obstrutiva (cistite idiopática felina obstrutiva).

Grupo de cistite bacteriana (GCB): Composto por sete gatos (quatro machos e três fêmeas), com histórico de DTUIF e com infecção bacteriana vesical no momento da avaliação.

Os resultados foram analisados por meio de estatística descritiva e análise de variância para dados não pareados (One-way ANOVA), seguida pelo teste de Tukey para comparação de médias $(\alpha=0,05)$. Todas as análises e gráficos foram realizados por meio do programa "Graphpad Prism version 6.00 for Windows", Graphpad Software, San Diego, Califórnia, USA.

\section{RESULTADOS E DISCUSSÃO}

As características gerais dos 50 animais, distribuídos nos respectivos grupos, como raça, comprimento do pelo, idade, e condição das gônadas estão descritas, com as respectivas frequências de ocorrência nas Tabelas 1 e 2. Observou-se que 37 (74\%) gatos não tinham raça definida e os restantes eram das raças Persa (6;12\%), Siamês (6; 12\%) e Angora (1; 2\%). Quanto ao sexo, $38(76 \%)$ eram machos e 12 $(24 \%)$ eram fêmeas. As idades dos pacientes variaram de um a 11 anos e os pesos corporais variaram de $2,9 \mathrm{~kg}$ a $7,4 \mathrm{~kg}$. Quanto às condições das gônadas, do total de 12 fêmeas, 11 $(92 \%)$ eram castradas e dos 38 machos, 30 (79\%) eram castrados.
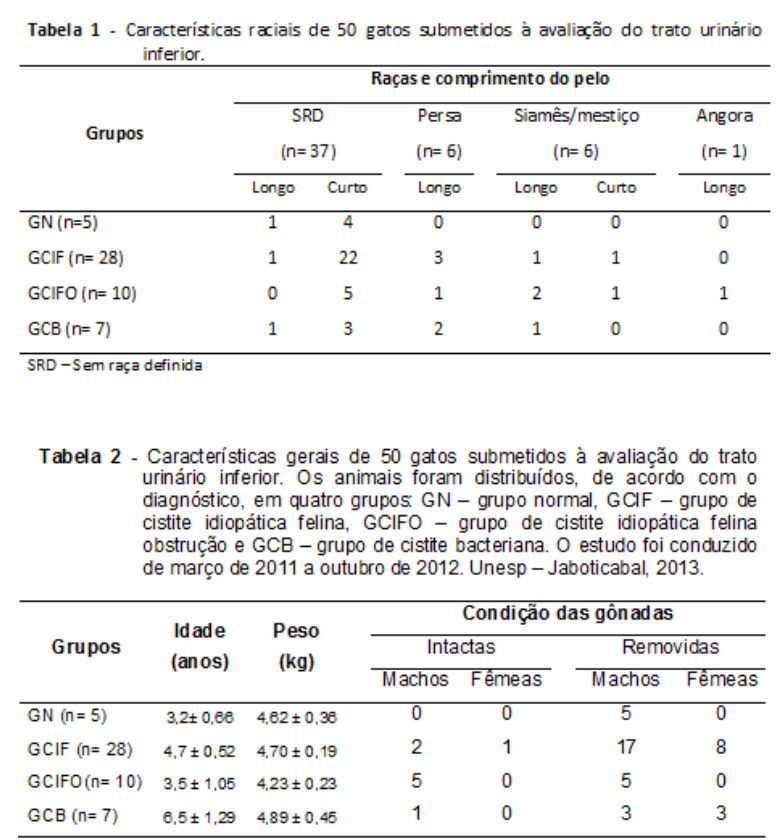

Com relação à qualidade e à frequência da dieta, houve predomínio das rações Super Premium e Premium e do oferecimento ad libitum (Tabela 3). A ração do tipo Super Premium era usada como alimento para 22 (44\%) dos gatos, a ração Premium para 19 (38\%) dos gatos e a ração do tipo Econômica para nove $(18 \%)$ dos gatos. Observou-se que todos recebiam alimento seco e apenas dois animais faziam uso de dieta úmida como complemento da dieta seca. Os dois gatos com completo de dieta úmida (GCIF), um macho e uma fêmea, apresentaram densidade urinária 1,050 e 1,047, respectivamente, destacandose que a média do grupo foi de (1,045 \pm 0,002). 


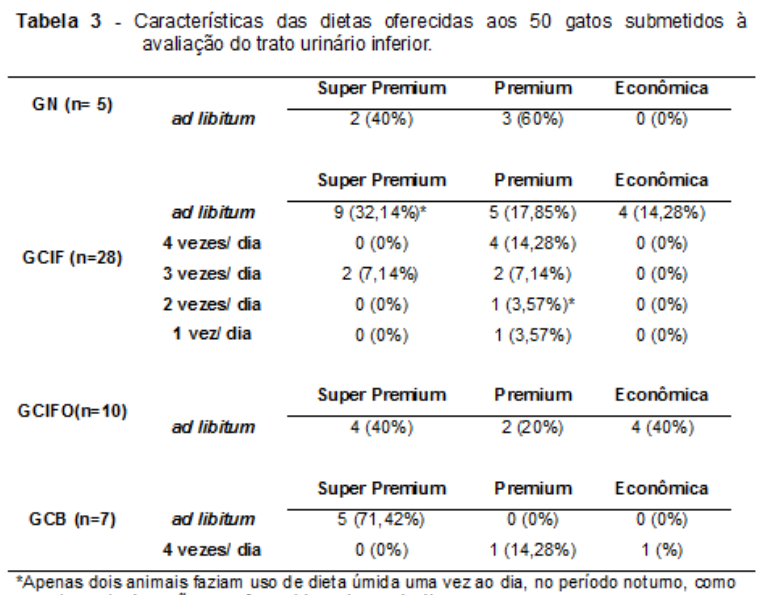

complemento da raçẫo seca fom ecida ao longo do dia.

A frequência de histórico de SDTUI em ocasiões anteriores foi maior no GCIF $(89,3 \%)$ e no GCB $(85,7 \%)$ do que no GCIFO (40\%). Trinta (67\%) do total de 45 animais doentes estavam em crise no momento do atendimento. Destaca-se que todos os gatos com GCIFO estavam em crise. O uso de antibioticoterapia em algum momento durante episódios anteriores de SDTUI foi relatado em 17 (38\%) casos, sendo que, 16 pacientes receberam enrofloxacina e um recebeu penicilina (Tabela 4).

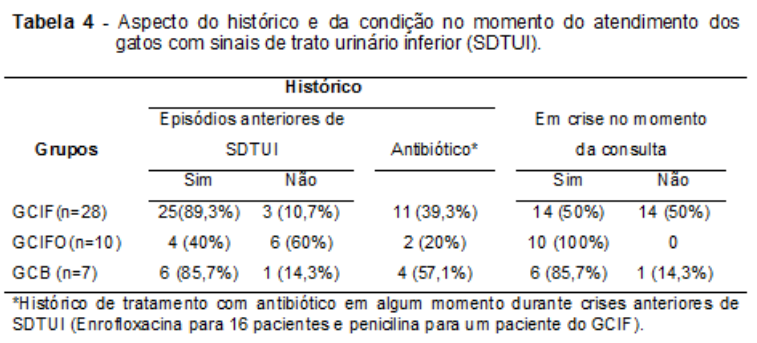

Os resultados das urinálises (densidade urinária e sedimento urinário), razão proteína/creatinina urinária (UPC) e exame microbiológico, estão distribuídos em função dos grupos, na Tabela 5. Foram observadas urinas com concentrações acentuadas (densidade urinária $\geq 1,035$ ) na maioria dos animais do GN (80\%) e GCIF $(71,4 \%)$. No GCB predominaram $(57,1 \%)$ as concentrações moderadas (densidade urinária $1,012>1,035)$. A avaliação do sedimento urinário revelou que, além das amostras de urina dos gatos sadios, não houve alteração em três amostras do GCIF, uma do GCIFO e uma do GCB. A hematúria foi o achado predominante nas amostras dos gatos doentes; cristalúria ocorreu em somente $25 \%$ das amostras do GCIF e $33 \%$ das amostras do GCIFO; e a leucocitúria (piúria) foi verificada em $57 \%$ das amostras do GCB, 33\% do GCIFO e $13 \%$ do GCIF.

Os resultados de UPC indicaram proteinúria em duas amostras do GN e em três amostras do GCIF. Resultados inconclusivos de UPC, ou seja, sujeitos a confirmação por repetições, ocorreram em duas amostras do GCIF e três do GCB (Tabela 5).

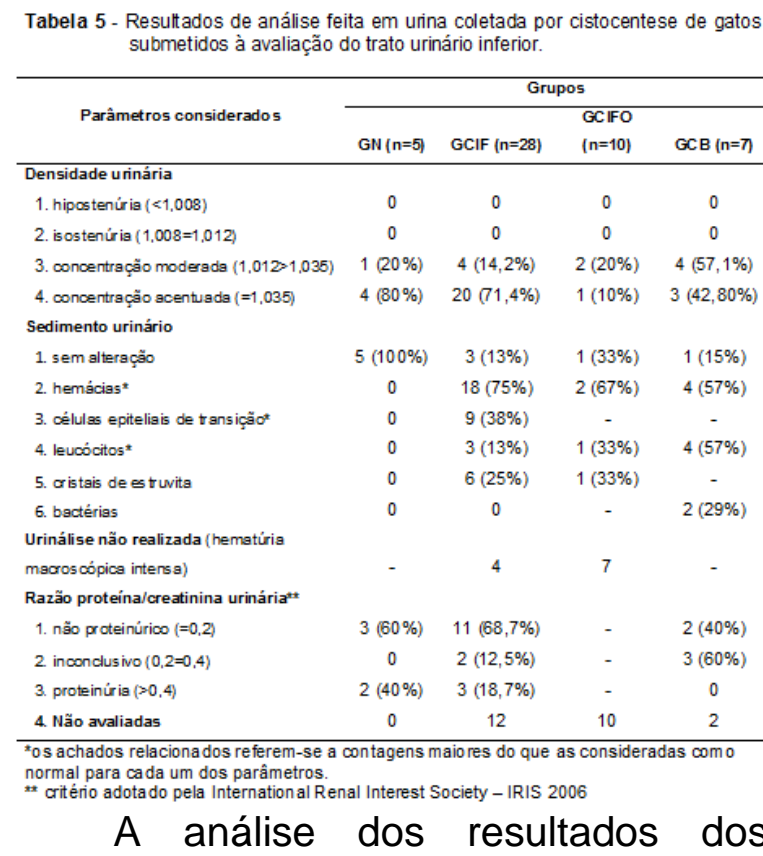
parâmetros urinários indicou que a média de densidade do GCIF foi significativamente maior que a do GCB e que as médias dos valores de $\mathrm{pH}$ e UPC não variaram significativamente entre os grupos (Tabela 6).

A bioquímica sérica revelou concentrações de ureia e creatinina acima dos valores de referência, para os gatos do GCIFO. Ambas as médias foram significativamente maiores que as respectivas médias dos gatos do GN, GCIF e GCB, evidenciando a associação com o quadro obstrutivo (Tabela 7). Os resultados dos hemogramas dos pacientes foram 
considerados normais em todos os grupos.

\begin{tabular}{|c|c|c|c|c|c|}
\hline & GN & GaF & GCIFO & GCB & $\begin{array}{l}\text { FValores de } \\
\text { referência }\end{array}$ \\
\hline Densidade & $1,043 \pm$ & $1,045 \pm$ & $1,031 \pm$ & $1,033 \pm$ & \\
\hline urinária & $0,004^{13}$ & $0,002^{2}$ & $0,007^{12}$ & $0,003^{8}$ & $1,002-1,070$ \\
\hline $\mathrm{pH}$ urinário & $6,1 \pm 0,10^{2}$ & $6,38 \pm 0,15^{2}$ & $5,50 \pm 0,29^{2}$ & $6,29 \pm 0,18^{2}$ & $5-9$ \\
\hline UPC & $0,23 \pm 0,10^{2}$ & $0,26 \pm 0,06^{2}$ & . & $0,24 \pm 0,05^{2}$ & $=0,2$ \\
\hline
\end{tabular}

Tabela 7 - Concentrações séricas de ureia e creathina (média \pm erro padrão da média).

\begin{tabular}{|c|c|c|c|c|c|}
\hline & GN & GCIF & GCIFO & GCB & $\begin{array}{l}\text { \#Valores de } \\
\text { referência }\end{array}$ \\
\hline & $55,61 \pm$ & $54,03 \pm$ & $192,58 \pm$ & $48,41 \pm$ & \\
\hline Ureia (mg/dl) & $4,52^{*}$ & $4,81^{\circ}$ & $67, \epsilon^{8}$ & $4,16^{*}$ & $32-75$ \\
\hline \multicolumn{6}{|l|}{ Creatinina } \\
\hline$(\mathrm{mg} / \mathrm{dl})$ & $1,60 \pm 0,04^{*}$ & $1,44 \pm 0,04^{*}$ & $6,02 \pm 1,64^{\circ}$ & $1,58 \pm 0,12^{*}$ & $0,8-2,0$ \\
\hline
\end{tabular}

Quanto aos achados da ultrassonografia vesical (conteúdo com sedimento; espessura e regularidade de parede), os gatos do GN não tiveram alteração. No GCIF houve predominância (66\%) de gatos com pouca quantidade de sedimentos (alteração leve) e apenas um paciente apresentou espessamento leve de parede vesical. Dos cinco gatos do GCB que foram avaliados, três tinham alteração leve do sedimento, um tinha sedimento abundante (intenso) e um não tinha alteração e a parede vesical estava espessada em três animais e normal em outros dois. Os gatos do GCIFO não foram avaliados por ultrassonografia, uma vez que os procedimentos para desobstrução uretral foram priorizados.

No grupo de cistite bacteriana foram isolados Escherichia coli em 4 $(57,1 \%)$ e Staphylococcus sp em 3 $(42,8 \%)$ das amostras. À sedimentoscopia das amostras de urina do GCB, o resultado foi normal em apenas um gato e houve alterações de intensidades variáveis de hematúria, piúria e bactériúria nos demais (Tabela 8).

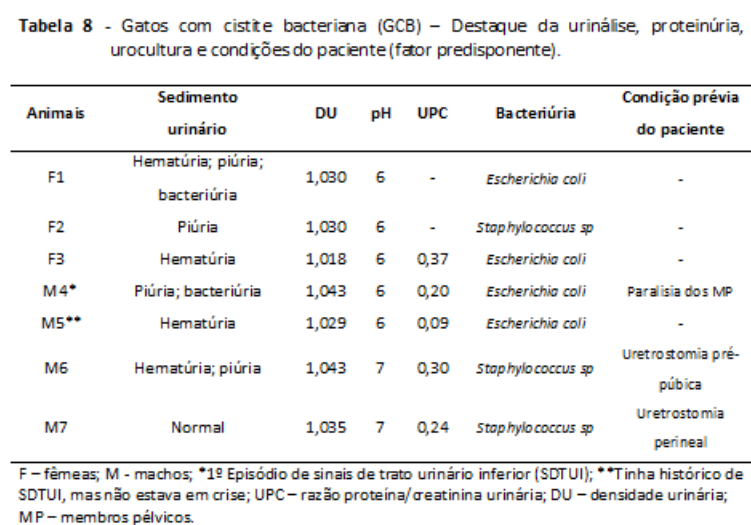

Há relatos de que gatos de raças puras são mais predispostos à DITUIF, em especial as raças Persa e Himalaia (WILLEBERG, 1984; LEKCHAROENSUK et al., 2001), enquanto gato Siamês (pelo curto) tem risco menor para DITUIF (LEKCHAROENSUK et al., 2001). BUFFINGTON et al. (2006), entretanto, afirmaram que a DITUIF não tem relação com o tamanho do pelo e nem com a raça do gato, pois há muitas razões para ocorrer diferenças entre os resultados de estudos epidemiológicos realizados em locais e momentos diferentes, com quantidade de animais, critérios utilizados e raças variadas, o que limita a comparação. No presente estudo houve o predomínio de gatos sem raça definida, provavelmente porque estes predominam dentre os felinos atendidos pelo SNUV, corroborando a afirmação de BUFFINGTON et al. (2006).

A associação do consumo de dieta seca com a ocorrência de CIF tem sido relatada há várias décadas (OSBORNE et al., 1995; BUFFINGTON et al., 1997; JONES et al., 1997; MARKWELL et al., 1998; MARKWELL et al.,1999; SÆVIK et al., 2011). Diversos estudos indicam que a ingestão maior de água, que pode ser obtida por meio do consumo de alimentos enlatados, pode prevenir a ocorrência de episódios de SDTUI (MARKWELL et al., 1999), pois resulta em diminuição da densidade e da concentração de substâncias nocivas na urina (WESTROPP, 2011). No presente 
estudo a alimentação de todos os gatos era feita com ração seca. Porém, deve ser salientado que a ração seca constitui dieta padrão na região onde vivem os gatos avaliados, em função da praticidade e custo acessível.

Em diversos estudos clínicos de gatos com CIF, com ou sem obstrução uretral, os percentuais de históricos de recorrência de SDTUI relatados foram de 56\% (DEFAUW et al., 2011) e 69\% (GERBER et al., 2005). Considerando somente gatos com obstrução por causas idiopáticas, tampões ou urólitos, GERBER et al. (2008) verificaram que 44\% apresentavam históricos de recorrências de SDTUI. No presente estudo, corroborando a literatura, o histórico de recorrência de SDTUI totalizou $77 \%$.

Dentre as avaliações do paciente com DTUIF, a urinálise pode trazer informações importantes que levam diretamente ao diagnóstico mais provável ou dar base para a decisão por outras provas e testes. Relata-se uma associação entre infecção urinária e densidade urinária $<1,020$ em gatos (BAILIFF et al., 2006). Contudo, BAILIFF et al. (2008) afirmam que não há associação entre diminuição da densidade urinária e resultado positivo de cultura de urina, e existe correlação positiva entre a severidade dos achados sedimentoscópicos (piúria, bacteriúria e hematúria) e cultura de urina positiva. Os resultados aqui apresentados mostram que a média de densidade urinária foi significativamente menor no GCB $(1,033 \pm 0,003)$ em relação ao GCIF, mas não em relação ao GN e ao GCIFO, corroborando Bailiff et al. (2008).

Sabe-se que a prevalência de cristalúria em gatos com CIF não difere significativamente da prevalência em gatos normais. (RICH \& KIRK, 1969; KRUGER et al., 1991; KALKSTEIN et al., 1999; KRUGER \& OSBORNE, 2009; DEFAUW et al., 2011). Neste estudo observou-se cristalúria somente em $25 \%$ das amostras do GCIF e $33 \%$ das amostras do GCIFO. Um fator importante a ser ressaltado, refere-se ao fato de que cada amostra de urina, coletada neste estudo foi analisada em laboratório adjacente à sala de coleta, dentro de, no máximo, 30 minutos, depois de arrefecer sobre bancada. De acordo com relatos de Bartges \& Kirk (2009) a grande quantidade de cristalúria em gatos com SDTUI pode decorrer da demora do processamento das amostras de urina não acondicionadas adequadamente. $\mathrm{Na}$ bexiga não há variação da temperatura da urina, entretanto, após a coleta (in vitro), amostras submetidas a temperaturas baixas tendem à precipitação de cristais.

Defauw et al. (2011) relataram que em gatos machos com CIF não obstrutiva a média de UPC foi $0,8 \pm 1,6$. Contudo, os resultados de UPC dos grupos GN, GCIF e GCB do presente estudo indicaram que não houve proteinúria na maioria dos pacientes e dentre os poucos casos com UPC $>0,2$, incluíram-se dois gatos sadios.

Os resultados de hemograma e bioquímica sérica são normais em gatos com DITUIF não obstrutiva (KALKSTEIN et al., 1999). Entretanto, nos casos de DITUIF obstrutiva ocorre aumento das concentrações séricas de creatinina e ureia em decorrência da supressão da filtração glomerular (SÆVIK et al., 2011). No presente estudo os resultados dos exames hematológicos foram normais para todos os grupos avaliados, no entanto, no GCIFO observou-se azotemia, corroborando os dados da literatura.

Achados ultrassonográficos não são específicos e não caracterizam CIF, mas talvez sejam úteis para distinguir as diferentes causas de DTUIF. Espessamentos difusos de parede vesical, identificados à ultrassonografia, são observados em casos crônicos com 
episódios múltiplos de CIF (GERBER et al., 2005; WESTROPP, 2011). No presente estudo houve mais destaque para os resultados de ultrassonografia do conteúdo vesical (sedimento), que é bastante inespecífico para diagnosticar CIF. A grande contribuição da ultrassonografia foi para a exclusão de outras causas de SDTUI, como apontado por Westropp (2011).

Dos 50 gatos avaliados foi possível realizar urocultura somente nos cinco animais sadios e em 23 com SDTUI. Destes, $30,4 \%$ (sete gatos) tiveram uroculturas positivas. Quatro (17,3\%) dos gatos com cistite bacteriana não tinham causas predisponentes detectáveis pelos exames realizados. Contudo, os outros três tinham condições predisponentes para ITU. Um macho sem histórico de SDTUI tinha paralisia de membros pélvicos. Outros dois haviam sido submetidos a uretrostomia em função de estenose uretral associada com episódios frequentes de CIFO. De fato, a uretrostomia perineal pode poupar os gatos das repetições de obstrução uretral, embora os SDTUI possam reaparecer (GERBER et al., 2008; RUDA \& HEIENE, 2012). Entretanto, as infecções bacterianas no trato urinário passam a ser frequentes e recorrentes em pacientes com uretrostomia (BASS et al., 2005).

Estudos recentes indicam que $E$. coli e Staphylococcus felis, além de Enterococcus faecalis são os uropatógenos mais comuns em gatos com SDTUI (EGGERTSDÓTTIR et al., 2007; LITSTER et al., 2007; SFEVIK et al., 2011). Dos sete gatos com a cistite bacteriana, quatro tinham infecção por E. coli e três por Staphylococcus spp.

Os achados do presente estudo assemelham-se às informações mais recentes, sobre a "doença do trato urinário inferior dos felinos", provenientes de países do hemisfério norte. Os dados obtidos evidenciam a realidade clínica e epidemiológica em nosso meio e são úteis para enfraquecer idéias errôneas, melhorando, assim, os cuidados médicos para os felinos.

\section{CONCLUSÃO}

O estudo sobre a apresentação clínica e laboratorial de gatos com SDTUI, dentre os encaminhamentos para check-up de rotina ou por apresentar algum sinal sugestivo da doença do trato urinário, permitiu concluir que a CIF pode acometer tanto machos como fêmeas, sendo o processo obstrutivo frequente em gatos machos. Os fatores como raça pura, comprimento do pelo, castração, obesidade e vida em ambiente residencial não são condições imperativas ou relevantes para a manifestação de CIF obstrutiva ou não obstrutiva. Não há dependência entre a existência de cristalúria de estruvita e a manifestação de SDTUI e o diagnóstico de cistite bacteriana em gatos requer urocultura.

\section{NOTAS INFORMATIVAS}

O presente estudo foi aprovado pela Comissão de Ética no Uso de Animais (protocolo no 004307/13) da Faculdade de Ciências Agrárias e Veterinárias da Unesp - campus de Jaboticabal - SP e compreendeu o período de março de 2011 a outubro de 2012.

\section{REFERÊNCIAS}

BAILIFF, N. L.; NELSON, R. W.; FELDMAN,E. C. et al. Frequency and risk factors for urinary tract infection in cats with diabetes mellitus.

Journal of Veterinary Internal Medicine, v.20, p.850-855, 2006 
BAILIFF, N. L.; WESTROPP, J. L.; NELSON, R. W. et al. Evaluation of urine specific gravity and urine sediment as risk factors for urinary tract infections in cats. Veterinary Clinical

Pathology, v.37, p.317-322, 2008.

BARTGES, J. W.; KIRK, C. A. Interpreting and Managing Crystalluria. In: BONAGURA, J. D. \& TWEDT, D. C. Current Veterinary Therapy XIV. Philadelphia: Saunders, 2009, cap. 185. p. 850-854.

BASS, M.; HOWARD, J.; GERBER, B. Retrospective study of indication for and outcome of perineal urethrostomy in cats. Journal of Small Animal Practice, v.46, n.5, p.227-231, 2005.

BUFFINGTON, C. A. T. Lower urinary disease in cats: new problems, new paradigms. Journal of Nutrition, v.124, p.2643-2651, 1994.

BUFFINGTON, C. A. T.; CHEW, D. J.; KENDALL, M. S. et al. Clinical evaluation of cats with nonobstructive urinary tract diseases. Journal of the American Veterinary Medical Association,v.210, n.1, p.46-50, 1997.

BUFFINGTON, C. A. T.; WESTROPP, J. L.; CHEW, D. J.; BOLUS, R. R. Risk factors associated with clinical signs of lower urinary tract disease in indoor-housed cats. Journal of the American Veterinary Medical Association, v.228, n.5, p.722-724, 2006.

DEFAUW, P. A. M.; VAN de MAELE, I.; DUCHATEAU, L.; et al. Risk factors and clinical presentation of cats with feline idiopathic cystitis. Journal of Feline Medicine and Surgery, v.13, n.12, p.967-975, 2011.

EGGERTSDÓTTIR, A.V.; LUND, H. S.; KRONTVEIT, R.; SØRUM, H. Bacteriuria in cats with feline lower urinary tract disease: a clinical study of 134 cases in Norway. Journal of Feline Medicine and Surgery. v.9, n.6, p.458-465, 2007.

GERBER, B.; BORETTI, F. S.; KLEY, S. et al. Evaluation of clinical signs and causes of lower urinary tract disease in European cats. Journal of Small animal Practice. v.46, n.12, p.571577, 2005.

GERBER, B.; EICHENBERGER, S.; REUSCH, C. E. Guarded log-term prognosis in male cats with urethral obstruction. Journal of feline Medicine and Surgery. v.10, p.16-23, 2008.
JONES, B. R.; SANSON, R. L.; MORRIS, R. S. Elucidating the risk factors of feline lower urinary tract disease. New Zealand Veterinary Journal. v.45, p.100-108, 1997.

KALKSTEIN, T. S.; KRUGER, J. M.; OSBORNE, C. A. Feline Idiopathic Lower Urinary Tract Disease. Part I. Clinical Manifestations.

Compendium on Continuing Education for the Practicing Veterinarian, Yardley, v.21, n.1, p. 15-36. 1999.

KALKSTEIN, T. S. Doença Idiopática do trato Urinário Inferior. In: LAPPIN, M. R. Segredo em medicina interna de felinos. São Paulo: Artmed, 2004. cap. 47, p. 273-281.

KRUGER, J. M.; OSBORNE, C. A.; GOYAL, S. M. et al. Clinical evaluation of cats with lower urinary tract disease. Journal of the American Veterinary Medical Association. v.199, n.2, p.211-216, 1991

KRUGER, J. M.; OSBORNE, C. A. Management of feline non obstructive idiopathic cystitis. In: BONAGURA, J. D. \& TWEDT, D. C. Current Veterinary Therapy XIV. Philadelphia: Saunders, 2009, cap. 205. p. 944-950.

LEKCHAROENSUK, C.; OSBORNE, C. A.; LULICH, J. P. Epidemiologic study of risk factors for lower urinary tract disease in cats. Journal of the American Veterinary Medical Association. v.218, n.9, p.1429-1435, 2001.

LITSTER, A.; MOSS, S. M.; HONNERY, M. et al. Prevalence of bacterial species in cats with clinical signs of lower urinary tract disease: Recognition of Staphylococcus felis as a possible feline urinary tract pathogen.

Veterinary Microbiology, v.121, p.182-188, 2007.

MARKWELL, P. J.; BUFFINGTON, C. T.; SMITH, B. H. E. The effect of diet on lower urinary tract diseases in cats. The Journal of Nutrition. v.128, n.12, p.2753-2757, 1998.

MARKWELL, P. J.; BUFFINGTON, C. A. T.; CHEW, D. J. et al. Clinical evaluation of commercially available urinary acidification diets in the management of idiopathic cystitis in cats. Journal of the American Veterinary Medical Association. v.214, n.3, p.361-365, 1999.

OSBORNE, C. A.; KRUGER, J. M.; LULICH, J. P.; POLZIN, D. J. Disorders of the feline lower urinary tract. In: OSBORNE, C. A. \& FINCO, D. $R$. Canine and feline nephrology and urology. Baltimo: Williams \& Wilkins, 1995, p. 625-680. 
OSBORNE, C. A.; KRUGER, J. M.; LULICH, J. P.; POLZIN, D. J. Feline urologic syndrome, feline lower urinary tract disease, feline interstitial cystitis: what's in a name? Journal of the American Veterinary Medical Association. v.214, n.10, p.1470-1480, 1999.

RICH, L. J.; KIRK, R. W. The relationship of struvite crystals to urethral obstruction in cats. Journal of the American Veterinary Medical Association. v.154, p.153, 1969.

RUDA, L.; HEIENE, R. Short- and long-term outcome after perineal urethrostomy in 86 cats with feline lower urinary tract disease. Journal of Small Animal Practice, Oxford, v.53, p.693 698, 2012.

SFEVIK, B. K.; TRANGERUD, C.; OTTESEN, N. et al. Causes of lower urinary tract disease in Norwegian cats. Journal of Feline Medicine and Surgery. v.13, n.6, p.410-417, 2011.

WESTROPP, J. L. Feline idiopathic cystitis. In: BARTGES, J. \& POLZIN, D. J. Nephrology and Urology of Small Animals, Ames: WileyBlackwell, 2011. cap. 75, p.745-754.

WILLEBERG, P. Epidemiology of naturally occurring feline urologic syndrome. Veterinary Clinics of North America Small Animal Practice. v.14, p.455-469, 1984. 